\title{
Lasers: The First Fifty Years
}

\author{
Robert W. Boyd,,$^{1, *}$ Ravindra Athale, ${ }^{2}$ Levent Onural, ${ }^{3}$ and Wolf Seka ${ }^{4}$ \\ ${ }^{1}$ The Institute of Optics, University of Rochester, Rochester, New York 14627, USA \\ ${ }^{2}$ Mitre Corporation, 7515 Colshire Drive, McLean, Virginia 22102, USA \\ ${ }^{3}$ Department of Electrical and Electronic Engineering, Bilkent University, Bilkent, Ankara TR-06800, Turkey \\ ${ }^{4}$ Laboratory for Laser Energetics, University of Rochester, Rochester, New York 14627, USA \\ *Corresponding author: boydrw@mac.com \\ Received 27 August 2010; accepted 27 August 2010; \\ posted 27 August 2010 (Doc. ID 134151); published 31 August 2010
}

\begin{abstract}
This year marks the 50th anniversary of the invention of the laser. The Optical Society of America is publishing this feature issue to celebrate this auspicious birthday. (C) 2010 Optical Society of America OCIS codes: $140.0140,140.3460,140.7240$.
\end{abstract}

This feature issue, Lasers: The First Fifty Years, deals with historical issues associated with the development of the laser and with current applications of the laser. A companion issue, Lasers: The Next Fifty Years, will be published later this year in the Journal of the Optical Society of America B and will emphasize the current status of laser performance and provide a prospectus of future developments in laser science and applications.

The early history of the laser can be summarized briefly as follows. Motivated by the success of the development of the maser both in the U.S. and in the Soviet Union, great interest developed in the possibility of constructing coherent light sources at much shorter wavelengths including the visible spectral region. A key step in this direction was the publication by Schawlow and Townes of a theoretical description of a procedure for constructing a maserlike device that operates at infrared and optical wavelengths [1]. Operating lasers were reported two years later in 1960. Here the story becomes somewhat complicated. Maiman is conventionally credited with operation of the first working laser. However, his published paper[2] from this era does not claim laser action, but only the narrowing of the emission spectrum of the gain medium. A later

0003-6935/10/250LF1-01 $\$ 15.00 / 0$

(C) 2010 Optical Society of America paper in the same year [3] from a group at Bell Laboratories provides much more compelling evidence of laser action based on the coherence properties of the emitted radiation. In any case, the laser was certainly born in 1960, and by the very next year was being used in scientific research, for example, in the creation of the field of nonlinear optics [4].

This feature issue begins with two review articles that summarize various aspects of the early history of the laser. It then presents several additional articles that describe various aspects of modern optical technology that are enabled by the availability of the laser. These articles serve to present a snapshot of the current status of laser science and engineering on the 50th anniversary of the laser.

\section{References}

1. A. L. Schawlow and C. H. Townes, "Infrared and optical masers," Phys. Rev. 112, 1940-1949 (1958).

2. T. H. Maiman, "Optical and microwave-optical experiments in ruby," Phys. Rev. Lett. 4, 564-566 (1960).

3. R. J. Collins, D. F. Nelson, A. L. Schawlow, W. Bond, C. G. B. Garrett, and W. Kaiser, "Coherence, narrowing, directionality, and relaxation oscillation in the light emission from ruby," Phys. Rev. Lett. 5, 303-305 (1960).

4. P. A. Franken, A. E. Hill, C. W. Peters, and G. Weinreich, "Generation of optical harmonic," Phys. Rev. Lett. 7, 118-119 (1961). 